\title{
The Healthy Teen Project: Tools to Enhance Adolescent Health Counseling
}

\author{
Ardis L. Olson, $M D_{i}{ }^{1}$ Cecelia A. Gaffney, MEd ${ }_{i}{ }^{1}$ Viking A. Hedberg, $M D, M P H_{i}{ }^{2}$ Wendy Gladstone, $M D_{i}{ }^{3}$ Sam Dugan, $M D_{i}{ }^{4}$ \\ Robert Mathes, $M D_{i}{ }^{5}$ Paul Reiss, $M D^{6}$ \\ 'Dartmouth Medical School, Hanover, NH \\ ${ }^{2}$ Plymouth Pediatrics, Dartmouth-Hitchcock Clinic, Plymouth, NH \\ ${ }^{3}$ Exeter Pediatric Associates, Exteter, NH \\ ${ }^{4}$ Bedford Pediatrics, Dartmouth-Hitchcock Clinic, Bedford, NH \\ ${ }^{5}$ Abenaki Family Physicians, Wolfeboro Falls, NH \\ ${ }^{6}$ Evergreen Family Health, Williston, Vt
}

Ann Fam Med 2005;3(Suppl 2):S63-S65. DOI: 10.1370/afm. 359.

Conflicts of interest: none reported

CORRESPONDING AUTHOR

Ardis L. Olson, MD, Clinicians Enhancing Child Health Network, Dartmouth Medical School, 7925 Rubin Bldg, One Medical Center Dr, Lebanon, NH 03756, ardis.olson@dartmouth.edu

\section{PURPOSE}

$\mathrm{T}$ The leading causes of preventable disease, disability, health care burden, and premature death in the United States are related to 4 health risk behaviors: sedentary lifestyle, unhealthy diet, tobacco use, and risky drinking. These behaviors are often interrelated, occur in a complex context and have their roots in childhood and adolescence.

Given the competing demands of primary care, only a very limited portion of the doctor-patient interaction is devoted to addressing these health risk behaviors. ${ }^{1}$ The visit is instead structured around gathering a health history and performing the physical examination. To assist adolescents in making healthy decisions, the emphasis of clinical encounters needs to be shifted from data-gathering to helping patients adopt and maintain healthy behaviors.

The purpose of our study was to create tools to improve health risk screening, counseling, and support for targeted behavior change among adolescents in primary care practices. We set out to create a package of tools that would facilitate integration of effective adolescent health behavior counseling into primary care practices. We aimed to create tools that would be easy to implement, inexpensive, and effective, and would be sustainable for use in practice.

\section{METHODS}

The Healthy Teens Project formed a working group of academic faculty and 6 physician innovators from 3 pediatric and 3 family practices in the Clinicians Enhancing Child Health $(\mathrm{CECH})$ practice-based research network at Dartmouth Medical School. The $\mathrm{CECH}$ network of 24 primary care practices in New Hampshire and Vermont is committed to improving clinical services for children and adolescents. For this project, we developed an adolescent health risk assessment tool (screener) administered on a personal digital assistant (PDA). The 90-item screener took 7 to 8 minutes for teenagers to complete and assessed nutrition, exercise, tobacco use, and alcohol use, as well as other health concerns and psychosocial risks included in the Guidelines for Adolescent Preventive Services. ${ }^{2}$ The physician reviewed a summary of the results on the PDA before the visit. Identification of teens' strengths and risks and concerns helped clinicians focus their discussion during the short time scheduled for the visit. We also created a health action plan form to record specific steps adolescents were willing to take to improve their health. Adolescents took this "health prescription" home. Those who committed to action steps were offered follow-up via e-mail to provide problemsolving and to support their actions.

The physician innovators, with the support of the research team, used their own practices as laboratories to test the PDA screener and action plan forms. A beta version of each tool was developed by the research team. The group conferred monthly, revising tools in response to experiences in the practices. The research team provided technological support for data collection and assisted with incorporating the tools into current office systems. A 2-hour program on motivational interviewing and goal setting provided training to the innovators on patient-centered counseling and developing action plans. An e-mail follow-up protocol was developed whereby medical students would contact 
the teens and provide support for their planned actions over 4 weeks.

This report covers the key lessons learned about these new clinical tools from informal interactions and a survey of clinicians $(n=15)$ in the 6 practices who used the PDA screener and the action plan materials. We analyzed exit questionnaires given to adolescents before $(n=67)$ and after $(n=98)$ implementation of the new tools to assess their perceptions of the quality of the visit and the topics discussed. Adolescents' views of the PDA screening and desired follow-up are based on these exit questionnaires.

\section{LESSONS LEARNED}

Our experience suggests that collaboration between academic researchers and primary care clinicians is the key to developing practical tools and office systems. The project was rooted in a collaborative process wherein family medicine and pediatric practice innovators worked closely with academic researchers. This team process facilitated rapid cycles of development and testing of the PDA screener and its implementation in the primary care practice laboratories. Physician innovators shared real-life experiences and practical strategies to counsel teens and to integrate the PDA into the office flow. The action plan worksheet was tested in clinical care and revised in 4 cycles by the team. Without this team process, we would not have been able to respond to the real-world clinical issues. These innovators also served the role of informing and engaging other clinicians in their practice. Developing practical practice systems is enhanced by the ongoing work of a joint academic-primary care team.

PDA screening was well received by teens and clinicians. The PDA screeners offered a novelty that engaged adolescents. Teens preferred the PDA to paper screeners or "being grilled by the clinician." The small screen size and the feature that answers disappeared from view meant teens could privately complete the PDA screener while sitting next to a parent in the examination or waiting room. Teens were candid and willing to share personal information via the PDA, and the PDA opened up conversation with adolescents about important issues not otherwise revealed. In a postvisit survey of 98 adolescents, $73 \%$ said the screener made it easier to discuss issues with their health care professional. Clinicians' feedback overall was very favorable, and all the clinicians have continued to use the PDA screeners in their routine care

Technology allowed centralized data collection in this study. Information delimited by the Health Insurance Portability and Accountability Act (HIPAA) was collected from the PDAs. Data were sent from each practice via the Internet to a CECH central computer daily. We ultimately succeeded in establishing this data synchronization of PDAs in 5 of our 6 practices. This system can be used for ongoing data collection for other projects. For the sixth practice, we exchanged PDAs via mail. We had the challenge of negotiating with information technology consultants at multiple small practices. Despite the emerging electronic medical record and use of the Internet, we found community practices lacked on-site expertise and often had older equipment not compatible with our software. Once established, however, synchronization of the PDA was simple enough to allow primary care office and nursing staff to routinely transmit data to the central office.

Our experience suggests that committing to a postvisit action plan is challenging for teens. Most adolescents were not prepared to think of "problems" and changing health risk behaviors during routine checkups, in contrast to visits for an identified chronic condition or specific complaint. Clinicians discussed health risks from the screener as well as the concerns raised by the teens. Our clinicians found it difficult, however, to develop specific planned action steps with teens, especially younger teens. When a teen identified a specific concern, clinicians were more able to problem-solve and develop a plan. The action plans were the entry into e-mail counseling support, and thus this tool was offered to few teens. Whereas only $6 \%$ to $7 \%$ of teens were interested in e-mail for follow-up information, $28 \%$ were interested in receiving additional information after the visit. The lack of interest in e-mail contact from the office, even before being offered as follow-up to action plans, makes this option of postvisit support less likely to work for many teens. Selective use of action plans for teens once they are interested in making a change may be better received. The discussion of specific strengths and health risks was well received by adolescents and may be built on by providing all adolescents with specific written information about their strengths and health risks with targeted take-home materials.

\section{CONCLUSIONS}

The PDA-based screener has been well received by both adolescents and clinicians and is a first step to increasing health counseling during adolescent well care. Adolescents' completion of the screener on a PDA is an efficient, confidential method to collect comprehensive health information at well visits. This approach informs clinicians before meeting with teens and allows clinicians to use their limited time counseling rather than data-gathering. Engaging adolescents in specific 
health action steps is challenging. Clinicians need more training in motivational counseling to influence adolescents. Adolescent-appropriate informational materials and referrals also need to be available. With further development, this innovative health screener could be an attractive tool for clinicians wanting to improve the adolescent health care they provide.

To read or post commentaries in response to this article, see it online at http://www.annfammed.org/cgi/content/full/3/Suppl_2/S63.

Key words: Primary health care; adolescents; health behavior; practicebased research network; behavioral/psychosocial; teenagers; computers, handheld

Submitted December 20, 2004; submitted, revised, February 22, 2005; accepted March 16, 2005.
Funding support: This project was supported by Prescription for Health, a national program of The Robert Wood Johnson Foundation with support from the Agency for Healthcare Research and Quality.

Acknowledgments: We would like to acknowledge our participating practices: The Bedford and Plymouth regional pediatric practices of Dartmouth-Hitchcock Medical Center in Lebanon, NH, Exeter Pediatric Associates in Exeter, NH, Abenaki Family Physicians in Wolfeboro Falls, NH, and Evergreen Family Health in Williston, Vt. Thanks to Cheryl Bush for her administrative expertise.

\section{References}

1. Klein JD, Wilson KN. Delivering quality care: adolescents' discussion of health risks with their providers. J Adolesc Health. 2002;30:190-195.

2. Elster AB, Kuznets NJ, eds. AMA Guidelines for Adolescent Preventive Services: Recommendations and Rationale. Baltimore, Md: Williams and Wilkins; 1994.

\section{Modular Lifestyle Intervention Tool: A Handheld Tool to Assist Clinicians in Providing Patient-Tailored Counseling}

Steven W. Heim, MD, MSPH ${ }_{i}{ }^{1,3}$ Moban Nadkarni, $M D_{i}{ }^{2}$ Lisa K. Rollins, $P b D_{i}{ }^{1}$ Jobn B. Schorling, $M D_{i}{ }^{2}$ David B. Waters, $P b D_{i}{ }^{1}$

Fern R. Hauck, MD, MS ${ }_{i}{ }^{1}$ Scott M. Strayer, MD, MPH $H^{1,3}$

${ }^{1}$ Department of Family Medicine, University of Virginia Health System, Charlottesville, Va

${ }^{2}$ Department of Internal Medicine, University of Virginia Health System, Charlottesville, Va

${ }^{3}$ Department of Health Evaluation Sciences, University of Virginia Health System, Charlottesville, Va

Ann Fam Med 2005;3(Suppl 2):S65-S67. DOI: 10.1370/afm.360.

Conflicts of interest: none reported

CORRESPONDING AUTHOR

Steven W. Heim, MD, MSPH, Department of Family Medicine, University of Virginia Health System, PO Box 800729, Charlottesville, VA 22908-0729, heims@virginia.edu

\section{PURPOSE}

A lthough proven health promotion interventions exist for effecting health behavior change, most physicians rarely go beyond simple advice or education when discussing health risk behaviors. ${ }^{1}$ The primary goal of our project was to develop and assess the effectiveness of a handheld computer clinical decision support tool, the Modular Lifestyle Intervention Tool (MLIT). The MLIT was designed to improve clinicians' ability to provide patient-tailored counseling at the point of care regarding tobacco use and unhealthy diet as it contributes to obesity and overweight.

\section{METHODS}

We created the MLIT by convening a multidisciplinary software development group (SDG) that included a family medicine researcher with experience in obesity, 2 general internists with expertise in smoking cessation and motivational interviewing (MI), and a clinical psychologist with expertise in MI. The group successfully adapted 2 clinical guidelines (the Public Health Service's Clinical Practice Guideline for Treating Tobacco Use and Dependence ${ }^{1}$ and the National Heart, Lung, and Blood Institute's Obesity Education Initiative $^{2}$ ) and the behavioral health theories of the 5 $A^{\prime} \mathrm{s}^{3}{ }^{3}$ the stages of change, ${ }^{4}$ and $\mathrm{MI}^{5}$ into a logical and navigable format. The SDG used an iterative process, meeting weekly for approximately 12, 1-hour sessions. The physical layout of the MLIT was represented using Microsoft PowerPoint (Microsoft Corp, Redmond, Wash), which visually simulated the spatial limitations of a handheld computer screen. Hypertext links within this program were used to simulate the navigation between screens on a handheld computer. Professional 